\title{
AN UNSOLVED PROBLEM ON THE POWERS OF $\frac{3}{2} *$
}

K. MAHLER

(Received 19 October 1966)

Let $\alpha$ be an arbitrary positive number. For every integer $n \geqq 0$ we can write

where

$$
\alpha\left(\frac{3}{2}\right)^{n}=g_{n}+r_{n},
$$

$$
g_{n}=\left[\alpha\left(\frac{3}{2}\right)^{n}\right]
$$

is the largest integer not greater than $\alpha\left(\frac{3}{2}\right)^{n}$, i.e. the integral part of $\alpha\left(\frac{3}{2}\right)^{n}$, and $r_{n}$ is its fractional part and so satisfies the inequality

$$
0 \leqq r_{n}<1 \text {. }
$$

We say that $\alpha$ is a $Z$-number if

$$
0 \leqq r_{n}<\frac{1}{2} \quad \text { for all suffixes } n \geqq 0 .
$$

Several years ago, a Japanese colleague proposed to me the problem whether such $Z$-numbers do in fact exist. I have not succeeded in solving this problem, but shall give here a number of incomplete results. In particular, it will be proved that the set of all $Z$-numbers is at most countable.

\section{1}

Assume that $\alpha$ is a $Z$-number. Evidently

$$
g_{n+1}+r_{n+1}=\frac{3}{2}\left(g_{n}+r_{n}\right) \text {. }
$$

Here $g_{n}$ and $g_{n+1}$ are integers, while $r_{n}$ and $r_{n+1}$ lie in the interval

$$
J=\left[0, \frac{1}{2}\right) \text {. }
$$

Hence one of the following two cases must hold.

(A) $g_{n}$ is an even number, hence $\frac{3}{2} g_{n}$ is an integer. Since

necessarily

$$
0 \leqq \frac{3}{2} r_{n}<\frac{3}{4}
$$

$$
g_{n+1}=\frac{3}{2} g_{n} \text { and } r_{n+1}=\frac{3}{2} r_{n} .
$$

- Presented as the 1966 Behrend Memorial Lecture at the University of Melbourne, 14 October 1966. 
(B) $g_{n}$ is an odd number and so both numbers $\frac{3}{2} g_{n} \mp \frac{1}{2}$ are integers. Since $\frac{3}{2} r_{n}+\frac{1}{2}$ cannot lie in $J$, we now must have

Put

$$
g_{n+1}=\frac{3}{2} g_{n}+\frac{1}{2} \text { and } r_{n+1}=\frac{3}{2} r_{n}-\frac{1}{2} .
$$

$$
\varepsilon_{n}= \begin{cases}0 & \text { if } g_{n} \text { is even, } \\ 1 & \text { if } g_{n} \text { is odd. }\end{cases}
$$

The two cases (A) and (B) can then be combined in the one formula

$$
g_{n+1}=\frac{3}{2} g_{n}+\frac{1}{2} \varepsilon_{n}, \quad r_{n+1}=\frac{3}{2} r_{n}-\frac{1}{2} \varepsilon_{n} .
$$

We also see that the case (A) can hold only if

and case $(B)$ if

$$
0 \leqq r_{n}<\frac{1}{3}
$$

$$
\frac{1}{3} \leqq r_{n}<\frac{1}{2}
$$

Hence $\varepsilon_{n}$ may also be defined by

$$
\varepsilon_{n}= \begin{cases}0 & \text { if } 0 \leqq r_{n}<\frac{1}{3} \\ 1 & \text { if } \frac{1}{3} \leqq r_{n}<\frac{1}{2}\end{cases}
$$

\section{2}

From (2), Since

$$
g_{0}=-\frac{1}{3} \varepsilon_{0}+\frac{2}{3} g_{1}, \quad g_{1}=-\frac{1}{3} \varepsilon_{1}+\frac{2}{3} g_{2}, \cdots, \quad g_{n-1}=-\frac{1}{3} \varepsilon_{n-1}+\frac{2}{3} g_{n} .
$$

$$
g_{0}+r_{0}=\left(\frac{2}{3}\right)^{n}\left(g_{n}+r_{n}\right)
$$

it follows from these equations that

$$
g_{0}=-\frac{1}{3}\left\{\varepsilon_{0}+\frac{2}{3} \varepsilon_{1}+\left(\frac{2}{3}\right)^{2} \varepsilon_{2}+\cdots+\left(\frac{2}{3}\right)^{n-1} \varepsilon_{n-1}\right\}+\left(\frac{2}{3}\right)^{n} g_{n}
$$

and similarly also

$$
r_{0}=+\frac{1}{3}\left\{\varepsilon_{0}+\frac{2}{3} \varepsilon_{1}+\left(\frac{2}{3}\right)^{2} \varepsilon_{2}+\cdots+\left(\frac{2}{3}\right)^{n-1} \varepsilon_{n-1}\right\}+\left(\frac{2}{3}\right)^{n} r_{n} .
$$

These equations can be generalised. For this purpose put

Then

$$
\alpha_{0}=\alpha \text { and } \alpha_{m}=\left(\frac{3}{2}\right)^{m} \alpha .
$$

$$
\left(\frac{3}{2}\right)^{n}\left(g_{m}+r_{m}\right)=\left(\frac{3}{2}\right)^{n} \alpha_{m}=\left(\frac{3}{2}\right)^{m+n} \alpha=g_{m+n}+r_{m+n},
$$

and it follows in analogy to (3) and (4) that for all suffixes $m$ and $n$,

$$
g_{m}=-\frac{1}{3}\left\{\varepsilon_{m}+\frac{2}{3} \varepsilon_{m+1}+\left(\frac{2}{3}\right)^{2} \varepsilon_{m+2}+\cdots+\left(\frac{2}{3}\right)^{n-1} \varepsilon_{m+n-1}\right\}+\left(\frac{2}{3}\right)^{n} g_{m+n}
$$

and

$$
r_{m}=+\frac{1}{3}\left\{\varepsilon_{m}+\frac{2}{3} \varepsilon_{m+1}+\left(\frac{2}{3}\right)^{2} \varepsilon_{m+2}+\cdots+\left(\frac{2}{3}\right)^{n-1} \varepsilon_{m+n-1}\right\}+\left(\frac{2}{3}\right)^{n} r_{m+n}
$$


The formula (6) for $r_{m}$ immediately implies a convergent series for this number. For all $r_{m+n}$ lie in the interval $J$, while the factor $\left(\frac{2}{3}\right)^{n}$ tends to zero as $n$ tends to infinity. It follows therefore that for all suffixes $m \geqq 0$,

$$
3 r_{m}=\varepsilon_{m}+\frac{2}{3} \varepsilon_{m+1}+\left(\frac{2}{3}\right)^{2} \varepsilon_{m+2}+\cdots
$$

and in particular,

$$
3 r_{0}=\varepsilon_{0}+\frac{2}{3} \varepsilon_{1}+\left(\frac{2}{3}\right)^{2} \varepsilon_{2}+\cdots .
$$

Here the convergence is in the sense of ordinary real analysis.

Consider next the formula (5) for $g_{m}$. The last term $\left(\frac{2}{3}\right)^{n} g_{m+n}$ of this formula is a rational number the numerator of which is divisible by at least the $n$-th power of 2 . In the so-called 2 -adic analysis in the rational number field one considers numbers as small if they are divisible by a high power of 2 in the numerator, and as large if such a power of 2 occurs in the denominator. In this 2 -adic sense the sequence of numbers $\left(\frac{2}{3}\right)^{n} g_{m+n}$ tends to zero as $n$ tends to infinity. We may therefore write

$$
-3 g_{m}=\varepsilon_{m}+\frac{2}{3} \varepsilon_{m+1}+\left(\frac{2}{3}\right)^{2} \varepsilon_{m+2}+\cdots \quad \text { in the 2-adic sense, }
$$

and in particular,

$$
-3 g_{0}=\varepsilon_{0}+\frac{2}{3} \varepsilon_{1}+\left(\frac{2}{3}\right)^{2} \varepsilon_{2}+\cdots \quad \text { in the } 2 \text {-adic sense. }
$$

It is rather interesting that the same series converges in two different senses and to two different limits.

From this we can already deduce the fact the set of all $Z$-numbers is at most countable. For if the integer $g_{0} \geqq 0$ is given, then, by $\S 1$, the corresponding sequence of integers $\varepsilon_{0}, \varepsilon_{1}, \varepsilon_{2}, \cdots$ is determined uniquely, and so, by (8), also the fractional part $r_{0}$. We may express this result as follows.

(11) For any given non-negative integer $g_{0}$ there exists at most one $Z$-number in the interval $\left[g_{0}, g_{0}+1\right)$, and this $Z$-number lies in fact in the first half $\left[g_{0}, g_{0}+\frac{1}{2}\right)$ of this interval.

\section{4}

Much more can be said about the possible $Z$-numbers and their integral parts $g_{0}$.

All the fractional parts $r_{m}$, where $r=0,1,2, \cdots$, lie by construction in the interval $J=\left[0, \frac{1}{2}\right)$. This means by (7) that for every suffix $m$ the inequality

$$
\varepsilon_{m}+\frac{2}{3} \varepsilon_{m+1}+\left(\frac{2}{3}\right)^{2} \varepsilon_{m+2}+\cdots<\frac{3}{2}
$$


is satisfied. In this set of inequalities each of the numbers $\varepsilon_{m}, \varepsilon_{m+1}, \varepsilon_{m+2}, \cdots$ can assume only either of the two values 0 or 1 .

It is then, firstly, immediately clear that for no $m$ simultaneously

For this would imply that

$$
\varepsilon_{m}=\varepsilon_{m+1}=1 \text {. }
$$

$$
\varepsilon_{m}+\frac{2}{3} \varepsilon_{m+1}+\left(\frac{2}{3}\right)^{2} \varepsilon_{m+2}+\cdots \geqq \frac{5}{3}>\frac{3}{2},
$$

contrary to (12). Therefore

$$
\text { if } m<n \text { and } \varepsilon_{m}=\varepsilon_{n}=1 \text {, then } n \geqq m+2 \text {. }
$$

From the inequalities (12) one can deduce restrictions on those suffixes $m$ for which simultaneously $\varepsilon_{m}=\varepsilon_{m+2}=1, \varepsilon_{m+1}=0$. We omit this discussion because no use will be made of the results so obtained.

\section{5}

Denote from now on by

$$
M=\left\{m_{1}, m_{2}, m_{3}, \cdots\right\}, \text { where } 0 \leqq m_{1}<m_{2}<m_{3}<\cdots,
$$

the set of all suffixes $m$ for which $\varepsilon_{m}=1$. Thus

$$
\varepsilon_{m}= \begin{cases}1 & \text { if } m \in M, \\ 0 & \text { if } m \notin M .\end{cases}
$$

In other words, $g_{m}$ is even or odd according as to whether $m$ is, or is not, an element of $M$.

Further put

so that all the $G_{k}$ are odd.

$$
G_{k}=g_{m_{k}} \quad(k=1,2,3, \cdots),
$$

On applying the equation (5) with

thus with

$$
m=m_{k} \text { and } m+n=m_{k+1},
$$

it follows that

$$
\varepsilon_{m}=1, \quad \varepsilon_{m+1}=\varepsilon_{m+2}=\cdots=\varepsilon_{m+n-1}=0,
$$

hence that

$$
G_{k}=-\frac{1}{3}+\left(\frac{2}{3}\right)^{m_{k+1}-m_{k}} G_{k+1},
$$

$$
G_{k+1}=\left(\frac{3}{2}\right)^{m_{k+1}-m_{k}-1} \frac{3 G_{k}+1}{2} .
$$

This formula leads to the following algorithm connected with our problem. 
We shall use the notation

$$
\mathbf{2}^{a}|| H
$$

to denote that $H$ is divisible by $2^{a}$, but not by $2^{a+1}$.

\section{6}

Put

$$
a_{k}=m_{k+1}-m_{k}-1, \quad H_{k}=\frac{3 G_{k}+1}{2} .
$$

Then, by (14), the following properties hold.

For every $k \geqq 1$,

(16) $G_{k}$ is odd; $H_{k}$ is even; $a_{k} \geqq 1 ; 2^{a_{k}}|| H_{k}$; and $G_{k+1}=\left(\frac{3}{2}\right)^{a_{k}} H_{k}$ is odd.

Thus, starting with any odd integer $G_{1}$, these formulae allow to determine successively the integers

$$
H_{1}, a_{1} ; G_{2}, H_{2}, a_{2}: G_{3}, H_{3}, a_{3} ; \cdots
$$

If $G_{1}$ was the integral part of a $Z$-number, then this algorithm can be continued indefinitely. It thus provides a necessary (but not a sufficient) condition for $G_{1}$ to be the integral part of a $Z$-number.

By way of example, if we start with $G_{1}=13$, we obtain the following sequence of integers.

$$
\begin{array}{lll}
G_{1}=13 & H_{1}=20 & a_{1}=2 \\
G_{2}=45 & H_{2}=68 & a_{2}=2 \\
G_{3}=153 & H_{3}=230 & a_{3}=1 \\
G_{4}=345 & H_{4}=518 & a_{4}=1 \\
G_{5}=777 & H_{5}=1166 & a_{5}=1 \\
G_{6}=1749 & H_{6}=2624 & a_{6}=6 \\
G_{7}=29889 & H_{7}=44834 & a_{7}=1 \\
G_{8}=67251 & H_{8}=100877 . &
\end{array}
$$

Since $H_{8}$ is odd, the algorithm breaks off, and there is no $Z$-number between 13 and 14.

In spite of much computer work, no integer $G_{1}$ is known for which the algorithm does not break off. It is thus highly problematical whether there do in fact exist $Z$-numbers.

\section{7}

If the existence of $Z$-numbers is assumed, further properties of such numbers can be obtained. 
Let us deal with the possible frequency of $Z$-numbers! We have already seen that there can be at most one $Z$-number in each interval between consecutive integers $g$ and $g+1$ where $g \geqq 0$. Thus, for $x>0$, there are not more than $x+1 Z$-numbers between 0 and $x$. This estimate can now be replaced by a stronger one.

Let us first consider $Z$-numbers with odd integral parts, say with the integral part $G_{1}$. Put

so that by (16),

$$
b_{k}=a_{k}+1 \quad \text { and } \quad c_{k}=a_{k}-1 \quad(k=1,2,3, \cdots),
$$

By (15) and (16),

$$
b_{k} \geqq 2 \text { and } c_{k} \geqq 0 \text { for all } k \text {. }
$$

$$
G_{k}=-\frac{1}{3}+\left(\frac{2}{3}\right)^{b_{k}} G_{k+1} .
$$

On applying this equation repeatedly, we find that

$$
G_{1}=-\frac{1}{3}\left\{1+\left(\frac{2}{3}\right)^{b_{1}}+\left(\frac{2}{3}\right)^{b_{1}+b_{2}}+\cdots+\left(\frac{2}{3}\right)^{b_{1}+b_{2}+\cdots+b_{n}}\right\}+\left(\frac{2}{3}\right)^{b_{1}+b_{2}+\cdots+b_{n+1}} G_{n+2}
$$

Here

$$
B_{n}=-\frac{1}{3}\left\{1+\left(\frac{2}{3}\right)^{b_{1}}+\left(\frac{2}{3}\right)^{b_{1}+b_{2}}+\cdots+\left(\frac{2}{3}\right)^{b_{1}+b_{2}+\ldots+b_{n}}\right\}
$$

is a rational number with an odd numerator and with a denominator which is a power of 3 .

Let now $t$ be an arbitrarily large positive integer. For the given $Z$ number there exists just one suffix $n$ such that

$$
b_{1}+b_{2}+\cdots+b_{n} \leqq t<b_{1}+b_{2}+\cdots+b_{n+1} .
$$

There further is a unique integer $s_{n}$ satisfying

such that

$$
1 \leqq s_{n} \leqq 2^{t}-1
$$

$$
B_{n} \equiv s_{n}\left(\bmod 2^{t}\right),
$$

i.e. that the numerator of $B_{n}-s_{n}$ is divisible by $2^{t}$. It is then clear from (17) that also

$$
G_{1} \equiv s_{n}\left(\bmod 2^{t}\right)
$$

The rational number $B_{n}$, and so also the integer $s_{n}$, depend only on $t$ and on the ordered set of integers $b_{1}, b_{2}, \cdots, b_{n}$. Denote by $T(t)$ the number of ordered sets of integers $n, b_{1}, b_{2}, \cdots, b_{n}$ which satisfy the left-hand inequality (18). This number $T(t)$ is then also the number of all residue classes $s_{n}\left(\bmod 2^{t}\right)$ in which there can lie odd integral parts $G_{1}$ of $Z$-numbers. 
One can easily obtain an upper bound for $T(t)$. The left-hand inequality (18) is equivalent to the inequality

$$
c_{1}+c_{2}+\cdots+c_{n} \leqq t-2 n ;
$$

hence $T(t)$ may also be defined as the number of ordered solutions $n$, $c_{1}, c_{2}, \cdots, c_{n}$ of this inequality where now $c_{1}, c_{2}, \cdots, c_{n}$ may run independently over all non-negative integers. For each separate value of $n$, this inequality has

$$
\left(\begin{array}{c}
{[t-2 n]+n} \\
n
\end{array}\right)=\left(\begin{array}{c}
t-n \\
n
\end{array}\right)
$$

solutions, and hence, summing over $n$,

$$
T(t)=\left(\begin{array}{c}
t-1 \\
1
\end{array}\right)+\left(\begin{array}{c}
t-2 \\
2
\end{array}\right)+\left(\begin{array}{c}
t-3 \\
3
\end{array}\right)+\cdots
$$

where all terms after the $\left[\frac{t}{2}\right]$-th vanish.

This formula may be written as

$$
T(t)+1=\sum_{n=0}^{t}\left(\begin{array}{c}
t-n \\
n
\end{array}\right)=\sum_{n=0}^{t}\left(\begin{array}{c}
n \\
t-n
\end{array}\right) .
$$

By the binomial theorem, it implies that $T(t)+1$ is the coefficient of $z^{t}$ in the power series in powers of $z$ for

$$
\sum_{n=0}^{t}\{z(1+z)\}^{n}=\frac{1-\{z(1+z)\}^{t+1}}{1-z(1+z)}
$$

and hence $T(t)+1$ is also the coefficient of $z^{t}$ in the power series for

$$
f(z)=\frac{1}{1-z-z^{2}} .
$$

Put

$A=\frac{1+\sqrt{ } 5}{2}, \quad B=\frac{1-\sqrt{ } 5}{2}$, so that $A+B=1, \quad A B=-1, \quad A-B=\sqrt{ } 5$. Then

$$
1-z-z^{2}=(1-A z)(1-B z) \text { and } f(z)=\frac{1}{\sqrt{ } 5}\left(\frac{A}{1-A z}-\frac{B}{1-B z}\right) .
$$

On developing here $f(z)$ into a series in powers of $z$, it follows at once that

$$
T(t)=\frac{1}{\sqrt{ } 5}\left\{A^{t+1}-B^{t+1}\right\}-1 .
$$

Actually, $T(t)+1$ is the $(t+1)$-st term of the well known Fibonacci sequence. 
Since trivially $B^{t+1}$ has the limit 0 as $t$ tends to infinity, and since further $A<\sqrt{ } 5$, it also follows from (20) that, for sufficiently large $t$,

$$
T(t) \leqq\left(\frac{1+\sqrt{ } 5}{2}\right)^{t}
$$

By the definition of $T(t)$, there are $T(t)$ distinct residue classes $\left(\bmod 2^{t}\right)$ in which the integral part $G_{1}$ of a $Z$-number can lie when it is odd.

Consider next a $Z$-number $\alpha=g_{0}+r_{0}$ with even integral part $g_{0}$, say

$$
2^{m} \| g_{0} \text {. }
$$

Then

$$
\alpha, \frac{3}{2} \alpha,\left(\frac{3}{2}\right)^{2} \alpha, \cdots,\left(\frac{3}{2}\right)^{m} \alpha
$$

likewise are $Z$-numbers, and they have the integral parts

$$
g_{0}, \frac{3}{2} g_{0},\left(\frac{3}{2}\right)^{2} g_{0}, \cdots,\left(\frac{3}{2}\right)^{m} g_{0},
$$

respectively. Here $\left(\frac{3}{2}\right)^{m} g_{0},=G_{1}$ say, is an odd integer divisible by $3^{m}$, and

$$
g_{0}=\left(\frac{2}{3}\right)^{m} G_{1}, \quad \frac{3}{2} g_{0}=\left(\frac{2}{3}\right)^{m-1} G_{1}, \cdots, \quad\left(\frac{3}{2}\right)^{m} g_{0}=G_{1} .
$$

These $m+1$ products lie in the residue classes

$$
\left(\frac{2}{3}\right)^{\mu} G_{1}\left(\bmod 2^{t}\right)
$$

respectively, where $\mu$ runs over the successive values $\mu=m, m-1$, $m-2, \cdots, 1,0$. If $\mu \geqq t$, then $\left(\frac{2}{3}\right)^{\mu} G_{1}$ lies in the residue class $\equiv 0\left(\bmod 2^{t}\right)$.

Thus to every odd residue class $G_{1}\left(\bmod 2^{t}\right)$ containing the integral part of a $Z$-number there correspond at most $t$ even residue classes (22) in which there are likewise integral parts of $Z$-numbers.

(23) This implies that there cannot be more than

$$
(t+1) T(t)
$$

odd or even residue classes $\left(\bmod 2^{t}\right)$ containing the integral part of a $Z$ number.

Trivially,

$$
\frac{1+\sqrt{ } 5}{2}<2^{0.7}
$$

Thus, as soon as $t$ is sufficiently large, it follows from (21) that there exist at most 


$$
2^{0.7 \cdot t-1}
$$

odd or even residue classes $\left(\bmod 2^{t}\right)$ in which there is the integral part of at least one $Z$-number.

Denote now by $x$ a sufficiently large positive integer, and choose the integer $t$ such that

$$
2^{t} \leqq x-1<2^{t+1}
$$

Then every residue class $\left(\bmod 2^{t}\right)$ contains at most two integers $\leqq x-1$. Hence there can be at most two $Z$-numbers not greater than $x$ the integral parts of which lie in this residue class. By (23), the number of residue classes which need be considered is only

$$
2^{0.7 \cdot t-1}<\frac{1}{2} x^{0.7} .
$$

We obtain therefore the following result.

(24) For sufficiently large $x$ there are at most

$$
x^{0.7}
$$

Z-numbers satisfying

$$
0 \leqq \alpha \leqq x .
$$

This paper dealt with the numbers $\alpha$ for which the fractional parts $r_{n}$ defined in $\S 1$ satisfied the inequalities

$$
0 \leqq r_{n}<\frac{1}{2} \quad(n=0,1,2, \cdots) .
$$

It is possible to establish a similar theory if all the $r_{n}$ are assumed to lie in some other subinterval $\left[c, c+\frac{1}{2}\right)$ of $[0,1)$. It would be very interesting if a similar theory could be established for subintervals of smaller length, or perhaps even of arbitrarily small length.

Naturally, one can consider analogous problems for the products

$$
\alpha\left(\frac{p}{q}\right)^{n} \quad(n=0,1,2, \cdots)
$$

where $\alpha$ is again a positive number, and $p$ and $q$ are integers satisfying

$$
p>q \geqq 2, \quad(p, q)=1 .
$$

Institute of Advanced Studies

Australian National University

Canberra, A.C.T. 